\title{
Unsuspected aortic dissection in patient with diagnosis of prosthesis aortic valve dysfunction
}

\begin{abstract}
On March 2012 a 74-year-old woman was admitted to our hospital for acute pulmonary oedema. In 1994 she had undergone aortic valve replacement with a Bicarbon 23A and mitral valve repair (commissurotomy) for mitral valve stenosis Auscultation revealed normal prosthetic valve opening and closing clicks and a grade $2 / 6$ ejection systolic murmur. TTE showed an intermittent aortic prosthesis dysfunction with mean $(24 \mathrm{~mm} \mathrm{Hg})$ and peak $(100 \mathrm{~mm} \mathrm{Hg})$ aortic gradients. They were higher than previous values recorded one month before. While the diagnosis of an intermittent aortic prosthesis valve dysfunction was done, the patient was admitted to the operating theatre where she had an aortic and mitral valve replacement. An aortotomy was done and an intimal flap on the aortic wall was found. After the operation the patient had an uncomplicated recovery and she was discharged on her 7th postoperative day. Ten months later, on follow up, she was in good condition with no valve dysfunction.
\end{abstract}

\author{
Volume I Issue 6 - 2014
}

\section{Caterina Simon, Diego Cugola, Carlo Fino, Francesco Innocente, Maurizio Merlo, Amedeo Terzi, Lorenzo Galletti \\ Department of Cardiac Surgery, Papa Giovanni XXIII, Italy}

Correspondence: Caterina Simon, Department of Cardiac Surgery,Via OMS I, 24100 Bergamo, Italy, Tel 390352673560 , Email csimon@hpg23.it

Received: December 08, 2014 | Published: December 17, 2014

Keywords: ascending aorta, aortic dissection, aortic prosthesis, recovery, undergone aortic

\section{Case report}

A 74-year-old woman was admitted to our hospital for acute pulmonary oedema. She had undergone aortic valve replacement with a Bicarbon 23A 16years before because aortic stenosis caused by rheumatic valve disease and during the same operation she received a mitral valve repair (commissurotomy) for mitral valve stenosis. Until February 2008 her clinical condition was good. On February 2012 she was admitted in hospital for atrial flutter with high ventricular response. She was treated with amiodarone and than after 4 days she was discharge. Transthoracic echocardiography (TTE) was performed and showed a left ventricle with normal volume and diameter, with ipokinesia of the apex, EF $45 \%$, a normal aortic prosthesis motion, and a native mitral valve with mild stenosis. On March 2012 she was admitted again in our hospital for acute pulmonary oedema, auscultation revealed normal prosthetic valve opening and closing clicks and a grade 2/6 ejection systolic murmur. TTE showed an intermittent aortic prosthesis dysfunction with mean $(24 \mathrm{~mm} \mathrm{Hg})$ and peak $(100 \mathrm{~mm} \mathrm{Hg})$ aortic gradients, they were higher than previous values recorded one month before. Multiplan transesophageal echocardiography (TEE) was, therefore, performed and confirmed TTE data. Severe aortic valve prosthesis regurgitation occurred intermittently and than a pannus or a thrombus was suspected do to a partial occlusion of the leaflets. A cardiac catheterisation (CC) and a cinefluoroscopy (CF) were performed. On CC the coronary artery was without stenosis and the prosthesis valve was good. CF allowed proper visualization of the disks showing normal systolic motion and the prosthesis aortic valve has a good motion of the bileaflet (Figure 1). While the diagnosis of an intermittent aortic prosthesis valve dysfunction was done, the patients were admitted to the operating theatre where she had an aortic and mitral valve replacement. Sternotomy was performed and extracorporeal circulation was started. An aortotomy was done and no pannus or thrombus was seen on the prosthesis but an intimal flap on the aortic wall was found. So the diagnosis of chronic Stanford type A aortic dissection was done. The Aortic dissection involved only the ascending aorta and the proximal part of the aortic arch. We found two tears in this part of the ascending aorta and the descending was free of dissection. In the light of that we suppose that the dysfunction was intermittently why during some cardiac cycle the flap went into the prosthesis and performed the dysfunction with a high gradient stenosis. The aortic valve was replaced with an 23 Carpentier-Edwards prosthesis, and the mitral valve with a $\mathrm{n} 27$ Carpentier-Edwards prosthesis, and the aorta with a vascular prosthesis $\mathrm{n} 26$. The histological exam confirms the macroscopic diagnosis of chronic aortic dissection, a necrosis of the medial layer of the aortic wall was found. After the operation the patient had an uncomplicated recovery and she was discharged on her 7 th postoperative day. Ten months later, on follow up, she was in good condition with no valve dysfunction.

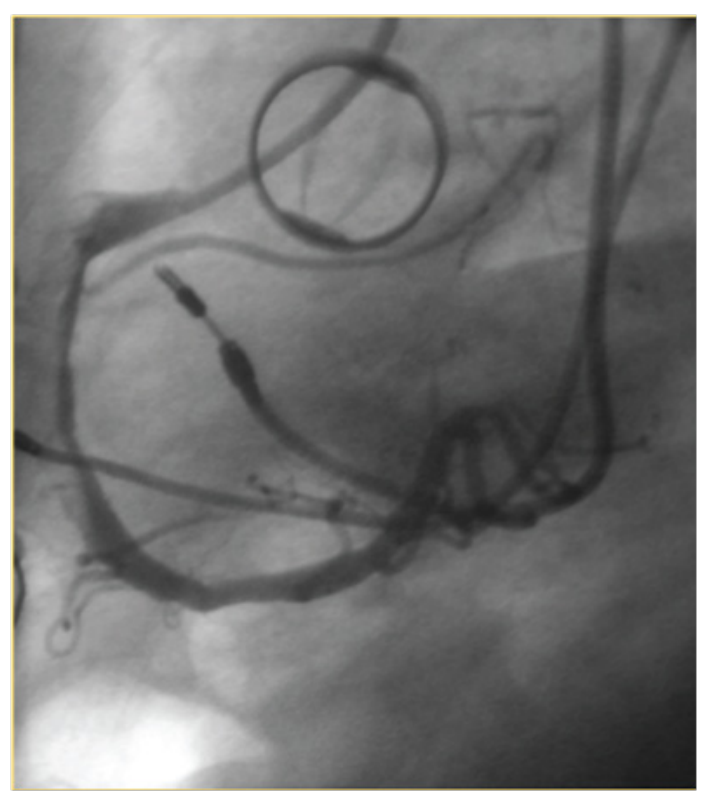

Figure I Cinefluoroscopic normal opening of the prosthetic valve. 


\section{Discussion}

The incidence of prosthetic valve obstruction was supposed to be from $0,1 \%$ to $4 \%$ per year, and is dependent on several factors including valve size, type and location, and adequacy of anticoagulation. ${ }^{1}$ Usual TTE, TEE, and CF are complementary methods to make a rapid, complete and correct diagnosis including valve morphology, presence of thrombus or pannus, and leaflet motion, but in this case they were not enough. We agree with Muratori et al. ${ }^{2}$ who observed that overall feasibility of leaflet motion analysis for aortic valve prosthesis was significantly less than in mitral valves for TTE and TEE. This was particularly true for patients with bileaflet valves, the most implanted prosthesis worldwide. Fluoroscopy permitted rapid and easy evaluation of mechanical prosthetic valve function, and in most cases allowed a distinction to be made between normal and dysfunctional prostheses. ${ }^{3}$ Intermittent alteration of mechanical leaflets in mitral prosthesis is a well-known phenomenon as a result of varying degrees of intermittent obstruction to the disk opening related to cyclic changes in atrial-ventricular gradients. On the contrary intermittent aortic dysfunction in mechanical aortic valves is a very rare condition and cyclic restriction of leaflet motion may not be easily explained by hemodynamic cyclic changes while it could be related to "pannus" interference that impedes normal closure of disks in an intermittent way. ${ }^{4,5}$ TEE was performed but it was not able to identify the intimal flap. In aortic prostheses, the overall feasibility of leaflet motion analysis was significantly less than in mitral valves. Anatomic and technical reasons make the alignment of the TEE ultrasound transducer with the moving parts of single or bileaflet prostheses difficult. As for mitral prostheses, a fairly good concordance of values between ultrasound and CF was detected. CF is superior to TTE in identifying disc motion. In the light of that we think that an Angio computer tomography must be performed, in case of aortic prosthesis intermittent dysfunction with an unclear aetiology.

\section{Acknowledgments}

None.

\section{Conflicts of interest}

Authors declare that there is no conflict of interest.

\section{References}

1. Barbetseas J, Nagueh S, Pitsavos C, et al. Differentiating thrombus from pannus formation in obstructed mechanical prosthetic valves: an evaluation of clinical. Transthoracic and transesophageal echocardiographic parameters. J Am Coll Cardiol. 1988;32(5):1410-1417.

2. Muratori M, Montorsi P, Terruzzi G, et al. Feasibility and Diagnosis accuracy of quantitative assessment of mechanical prostheses leaflet motion by transthoracic and transesophageal echocardiography in suspected prosthetic valve dysfunction. Am J Cardiol. 2006;97(1):94 100 .

3. Cianciulli TE, Lax Ja, Beck MA, et al. Cinefluoroscopic assessment of mechanical disc prostheses:its value as a complementary method to echocardiography. J Heart Valve Dis. 2005;14(5):664-673.

4. Karagiannis S, Karatasakis G, Sparagias K, et al. Intermittent acute aortic valve regurgitation: a case report of a prosthetic valve dysfunction. European J of Echocardiogr. 2008;9(2):291-293.

5. Mohr kahaly S, Kupferwasser I, Erbel R, et al. Value and limitations of transesophageal echocardiography in the evaluation of aortic prostheses. J Am Soc Echocardiogr. 1933;6(1):12-20. 